\title{
Do variations in the ITPA gene determine the risk of hepatitis $C$ virus relapse?
}
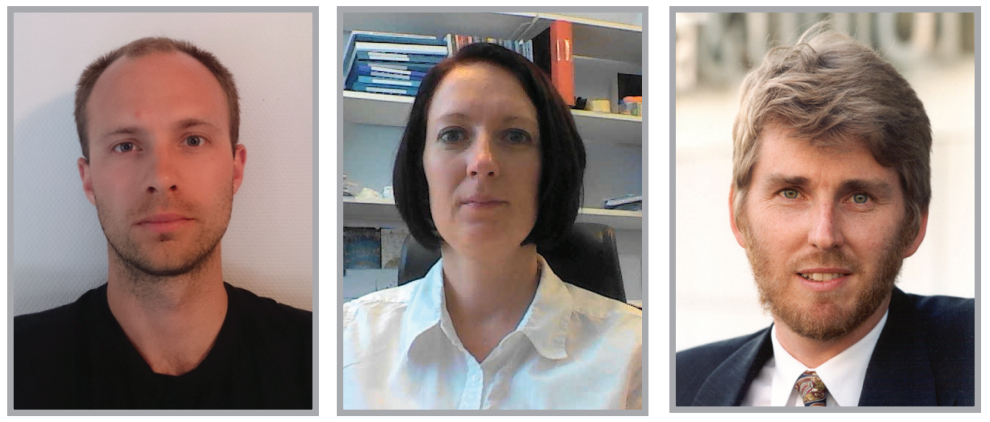

Jesper Waldenström', Kristina Nyström \& Martin Lagging ${ }^{*, 1}$

\section{ITPA polymorphisms}

\section{\& ribavirin-induced anemia}

A recent genome-wide association study demonstrated that two genetic variants, a missense variant in exon 2 (rs1127354, $\mathrm{P} 32 \mathrm{~T}$ ) and a splice-altering single-nucleotide polymorphism in intron 2 (rs7270101, IVS2) of the ITPA gene, entailing reduced ITPase activity, protecting against ribavirin-induced hemolytic anemia during interferon and ribavirin therapy for hepatitis $\mathrm{C}$ virus (HCV) genotype 1 [1]. This protection against anemia has been subsequently confirmed in numerous studies [2-8], but divergent results regarding the association with outcome have been reported.

\section{ITPA polymorphisms \& sustained virological response}

Several, but far from all studies have reported associations between homo- or hetero-zygosity at $\mathrm{A}_{\mathrm{rs} 127354}$ or $\mathrm{C}_{\mathrm{rs} 5270101}$, which are present in $37 \%$ of $\mathrm{HCV}$ patients [2], and a higher likelihood of achieving sustained virological response (sustained virological response [SVR]; i.e., undetectable HCV RNA 24 weeks after termination of therapy), mediated by reduced relapse rates [2-5]. This reduction in relapse resembles that reported when ribavirin was added to interferon, thus markedly improving outcomes as compared with prior interferon monotherapy [9]. In contrast to the aforementioned studies, others have not been able to demonstrate any significant association between ITPA variants and outcome $[1,6-8]$. These discordant results may potentially be explained by differences in adherence monitoring and ribavirin dosing.

When evaluating the impact of potential response predictors, monitoring of adherence to medication is essential in order to minimize the dilution effect caused by the inclusion in the analysis of patients not achieving SVR due to premature treatment termination secondary to neuropsychiatric side effects of interferon. Furthermore, the well-established relationship between reduced ITPase activity and protection against anemia may act as a confounding factor, as the latter subsequently improves ribavirin adherence [8], which consequently impacts on outcome.

When evaluating a cohort of northern European treatment-naive patients with

\section{KEYWORDS}

- hepatitis $C$ virus $\bullet$ inosine triphosphatase $\bullet$ inosine triphosphate pyrophosphatase - ITPA 
chronic HCV genotype 2/3 infection receiving peg-IFN- $\alpha 2 \mathrm{a} 180 \mu \mathrm{g}$ once weekly and the standard, lower ribavirin dose of $800 \mathrm{mg}$ daily (NORDynamIC study), a significant association between ITPA polymorphisms and treatment efficacy was noted, mediated by reduced relapse [2]. This observation could not be explained by improved adherence, protection against anemia or more efficient early elimination of the virus [10]. In the NORDynamIC study, adherence was closely monitored both by case report forms and patient diaries. The strong association between reduced ITPase activity and therapeutic outcome remained significant when patients were grouped by HCV genotype, IL28B or liver fibrosis. It was also noted in this study that reduced ITPase activity was significantly associated with lower plasma ribavirin levels [2], which are otherwise correlated with increased risk of treatment failure. Similarly, Clark et al. reported that among 70 genotype 2 or 3 -infected real-life clinic patients receiving $800 \mathrm{mg}$ ribavirin daily and $123 \mathrm{HCV}$ genotype 1 or 4 -infected treated with $1000-1200 \mathrm{mg}$ of ribavirin in combination with peg-IFN- $\alpha$, reduced ITPase activity was significantly associated with an increased likelihood of achieving SVR by means of reduced relapse [4]. However, in this study, the authors could not exclude improved adherence secondary to protection against anemia, even though reduced ITPase activity remained significantly associated with SVR despite the inclusion of ribavirin dose reductions and anemia in the multivariate analyses.

The aforementioned studies contrast with those by Thompson et al. [6] and Eskesen et al. [7], who also assessed HCV genotype 2/3-infected patients, finding that ITPA variants protected against anemia, but did not impact on SVR. In both of these studies, higher ribavirin dosing was used, (i.e., 1000/1200 mg/day [6] and $800-1400 \mathrm{mg} /$ day weight-based dosing [7]) instead of the conventional, lower 800-mg daily dose.

Similar to the observations noted among HCV genotype 2/3-infected patients, in a subset of 81 genotype $1 \mathrm{~b}$-infected Japanese patients with the favorable IL28B rs8099917 TT genotype treated with 600-1000-mg daily weightbased dosing of ribavirin and peg-IFN- $\alpha$ for 48-weeks, carriage of the minor $\mathrm{A}_{\mathrm{rs} 1127354}$ ITPA allele was associated with an improved likelihood of achieving SVR, as well as reduced relapse [3], possibly secondarily to improved ribavirin adherence. In another Japanese study among HCV genotype 1-infected patients treated with ribavirin $400-1000 \mathrm{mg} / \mathrm{day}$ and peg-IFN- $\alpha$ for 72 weeks because of late viral response, $A_{1127354}$ ITPA carriage was associated with a significantly higher likelihood of achieving SVR [5], again mediated by reduced relapse. In this latter study, both ribavirin concentration at week 44 and ITPA genotype were independently associated with SVR in a multivariate analysis [5].

By contrast, Holmes et al. observed among $546 \mathrm{HCV}$ genotype 1-infected patients receiving 1000-1200-mg weight-based ribavirin that the major ITPA variant, entailing normal ITPase activity, was associated with higher cumulative dose reduction, but not with ribavirin concentration at week 8 or SVR [8]. Thus, higher ribavirin dosing may possibly overshadow the impact of ITPA variants, as higher intracellular ribavirin levels may overcome the negative impact of normal ITPase activity.

\section{How can reduced ITPase activity have a ribavirin-like effect on relapse rates?}

ITPase is a highly conserved enzyme that recycles purines by converting ITP into IMP, thus protecting against the accumulation of rogue nucleotides (e.g., ITP and dITP), which otherwise may lead to erroneous incorporation into RNA and DNA, resulting in mutations, recombinations, altered RNA structures, irregular proteins and altered ATP-dependent signaling [11]. The consequences of ITPase deficiency remain largely unclear; however, it has been associated with increased drug toxicity when treating with purine analogs $[12,13]$.

Thus far, the underlying mechanism of action through which lower ITPase activity protects against relapse remains unclear; however, it possibly differs from that suggested regarding protection against ribavirin-induced anemia (i.e., diminished oxidative stress in erythrocytes through the evasion of ATP reduction) [14]. By contrast, reduced ITPase activity has been hypothesized to have a ribavirin-like mechanism of action [2]. In part, the antiviral effects of ribavirin, which also has little effect on viral load during HCV treatment [15] but markedly reduces relapse after discontinuation of therapy [16], are assumed to be achieved by inhibition of IMPDH, leading to depletion of GTP and a circuitous increase of ITP via the induction of IMP [17]. This parallels findings in genetic knockout models, where ITP concentrations surge as a consequence of a lack of ITPase [18], 
whereas intracellular levels of IMP (the substrate of IMPDH) diminish, which in turn results in lower concentrations of GTP. Therefore, it may be hypothesized that increased intracellular concentrations of ITP and decreased levels of GTP, whether as a result of lower ITPase activity or the extrinsic addition of ribavirin, lead to increased incorporation of this noncanonical nucleotide [11] by the nonstringent HCV RNA-dependent RNA polymerase, which lacks proofreading capacity, with ensuing random mutagenesis. This mechanism could potentially be augmented further by a similar incorporation of ribavirin triphosphate. Supportive of this notion is the reported strong synergistic effect of the inhibition of both the IMPDH and ITPA homologs in Escherichia coli and Saccharomyces cerevisiae leading to increased dITP and ITP levels in DNA and RNA [19]. In addition, inosine-containing RNA has been proposed to act as a potential danger signal, triggering the activation of both adaptive and innate immune responses [20].

\section{Possible clinical applications in HCV \& other viral infections}

There are potential clinical applications of determining ITPA genetic variants (e.g., lower daily dosing of ribavirin might be sufficient for HCV-infected patients with polymorphisms that encode reduced ITPase activity when receiving IFN-based therapy, whereas higher dosing might be preferential for patients who are homozygous for both major alleles with normal ITPase activity). This could affect future $\mathrm{HCV}$ treatment guidelines, even in more affluent regions in spite of the therapeutic improvements secondary to the recent introduction of the relatively costly direct-acting antiviral agents. Indeed, the majority of patients have normal ITPase activity and so may potentially benefit from ribavirin addition to some of the new IFN-free treatment regimens for $\mathrm{HCV}$. Similarly, wild-type ITPA carriers may also gain more from the use of ribavirin against other viral infections (e.g., respiratory syncytial virus, hepatitis E virus, Lassa fever and polio, among others). In addition, pharmacological inhibition of ITPase activity could also potentially mitigate the need for ribavirin, and thus be an innovative target for drug development.

\section{Conclusion}

ITPA polymorphisms entailing reduced ITPase activity are associated with an improved likelihood of achieving SVR resulting from the reduced risk of relapse following IFN and ribavirin combination therapy for $\mathrm{HCV}$ infection, and this impact on outcome is possibly more pronounced when lower ribavirin dosing is used. The underlying mechanism of this remains to be elucidated, but the incorporation of the potentially mutagenic, immunostimulatory and structure-altering ITP into the viral genome by less stringent viral polymerases lacking proofreading capabilities offers an intriguing hypothesis. This could potentially reveal a novel feature of innate immunity in addition to being an innovative target for future drug development. Thus, in conclusion, the impact of ITPA polymorphisms on $\mathrm{HCV}$ and other viral infection warrants further study.

\section{Financial \& competing interests disclosure}

Grants from the Swedish Research Council (Diarienr 2011:3821) and ALF Funds to the Sablgrenska University Hospital (Diarienr ALFGBG-143271) supported this work. The authors have no other relevant affliations or financial involvement with any organization or entity with a financial interest in or financial conflict with the subject matter or materials discussed in the manuscript apart from those disclosed.

No writing assistance was utilized in the production of this manuscript.

\section{References}

1 Fellay J, Thompson AJ, Ge D et al. ITPA gene variants protect against anaemia in patients treated for chronic hepatitis C. Nature 464(7287), 405-408 (2010).

2 Rembeck K, Waldenstrom J, Hellstrand K et al. Variants of the inosine triphosphate pyrophosphatase gene are associated with reduced relapse risk following treatment for HCV genotype 2/3. Hepatology 59(6), 2131-2139 (2014).
3 Kurosaki M, Tanaka Y, Tanaka K et al. Relationship between polymorphisms of the inosine triphosphatase gene and anaemia or outcome after treatment with pegylated interferon and ribavirin. Antivir. Ther. 16(5), 685-694 (2011).

4 Clark PJ, Aghemo A, Degasperi E et al. Inosine triphosphatase deficiency helps predict anaemia, anaemia management and response in chronic hepatitis $\mathrm{C}$ therapy. J. Viral Hepat. 20(12), 858-866 (2013).
5 Hai H, Tamori A, Enomoto $\mathrm{M}$ et al. Relationship between inosine triphosphate genotype and outcome of extended therapy in hepatitis $\mathrm{C}$ virus patients with a late viral response to pegylated-interferon and ribavirin. J. Gastroenterol. Hepatol. 29(1), 201-207 (2014).

6 Thompson AJ, Santoro R, Piazzolla V et al. Inosine triphosphatase genetic variants are protective against anemia during antiviral therapy for HCV2/3 but do not decrease dose 
reductions of RBV or increase SVR. Hepatology 53(2), 389-395 (2011).

7 Eskesen AN, Melum E, Moghaddam A et al. Genetic variants at the ITPA locus protect against ribavirin-induced hemolytic anemia and dose reduction in an $\mathrm{HCV}$ G2/G3 cohort. Eur. J. Gastroenterol. Hepatol. 24(8), 890-896 (2012).

8 Holmes JA, Roberts SK, Ali RJ et al. ITPA genotype protects against anemia during peginterferon and ribavirin therapy but does not influence virological response. Hepatology 59(6), 2152-2160 (2014).

9 Reichard O, Norkrans G, Fryden A, Braconier JH, Sonnerborg A, Weiland O. Randomised, double-blind, placebocontrolled trial of interferon alpha-2b with and without ribavirin for chronic hepatitis $\mathrm{C}$. The Swedish Study Group. Lancet 351(9096), 83-87 (1998).

10 Lagging M, Rembeck K, Waldenstrom J et al. ITPA variants are associated with reduced relapse rate but not with early virological responses hollowing $\mathrm{HCV}$ genotype 2/3 therapy. Hepatology doi:10.1002/hep.27108 (2014) (Epub ahead of print).
11 Sumi S, Marinaki AM, Arenas M et al. Genetic basis of inosine triphosphate pyrophosphohydrolase deficiency. Hum. Genet. 111(4-5), 360-367 (2002).

12 Stocco G, Cheok MH, Crews KR et al. Genetic polymorphism of inosine triphosphate pyrophosphatase is a determinant of mercaptopurine metabolism and toxicity during treatment for acute lymphoblastic leukemia. Clin. Pharmacol. Ther. 85(2), 164-172 (2009).

13 Bierau J, Lindhout M, Bakker JA. Pharmacogenetic significance of inosine triphosphatase. Pharmacogenomics 8(9), 1221-1228 (2007).

14 Hitomi Y, Cirulli ET, Fellay J et al. Inosine triphosphate protects against ribavirin-induced adenosine triphosphate loss by adenylosuccinate synthase function. Gastroenterology 140(4), 1314-1321 (2011).

15 Rotman Y, Noureddin M, Feld JJ et al. Effect of ribavirin on viral kinetics and liver gene expression in chronic hepatitis C. Gut 63(1), 161-169 (2013).

16 Hiramatsu N, Oze T, Yakushijin T et al. Ribavirin dose reduction raises relapse rate dose-dependently in genotype 1 patients with hepatitis $\mathrm{C}$ responding to pegylated interferon alpha-2b plus ribavirin. J. Viral Hepat. 16(8), 586-594 (2009).

17 Gebeyehu G, Marquez VE, Van Cott A et al. Ribavirin, tiazofurin, and selenazofurin: mononucleotides and nicotinamide adenine dinucleotide analogues. Synthesis, structure, and interactions with IMP dehydrogenase. J. Med. Chem. 28(1), 99-105 (1985).

18 Behmanesh M, Sakumi K, Abolhassani N et al. ITPase-deficient mice show growth retardation and die before weaning. Cell Death Different. 16(10), 1315-1322 (2009).

19 Pang B, Mcfaline JL, Burgis NE et al. Defects in purine nucleotide metabolism lead to substantial incorporation of xanthine and hypoxanthine into DNA and RNA. Proc. Natl Acad. Sci. USA 109(7), 2319-2324 (2012).

20 Liao JY, Thakur SA, Zalinger ZB, Gerrish KE, Imani F. Inosine-containing RNA is a novel innate immune recognition element and reduces RSV infection. PLoS ONE 6(10), e26463 (2011). 